\title{
Técnicas terapéuticas endovasculares
}

Endovascular therapeutic techniques

J. I. Bilbao, A. Martínez de la Cuesta, P. Domínguez Echavarri, O. Cosín, L. Desloques, B. Zudaire

\section{RESUMEN}

Las técnicas percutáneas y endovasculares han demostrado su eficacia en el tratamiento de una gran variedad de patologías. Los avances en la imagen diagnóstica así como en el desarrollo de nuevos materiales han posibilitado la realización de nuevos procedimientos, impensables hace no mucho años. La irrupción de esta nueva forma de tratar a los pacientes ha tenido, tiene y tendrá, aún más, un claro impacto en el enfoque multidisciplinar de múltiples enfermedades

Palabras clave. Terapéutica endovascular. Patología vascular. Patología hepática.

\begin{abstract}
Percutaneous and endovascular techniques have shown their efficacy in the treatment of a great variety of pathologies. The advances in diagnostic imaging as well as the development of new materials have made it possible to carry out new procedures that were unthinkable not many years ago. The irruption of this new form of treating patients has had, is having, and will have a clear impact on the multidisciplinary approach to numerous diseases.
\end{abstract}

Key words. Endovascular therapeutics. Vascular pathology. Hepatic pathology.

An. Sist. Sanit. Navar. 2005; 28 (Supl. 3): 117-134.

Servicio de Radiología. Clínica Universitaria de Navarra. Pamplona.

\author{
Correspondencia: \\ J. I. Bilbao \\ Dpto. de Radiología \\ Clínica Universitaria \\ Avda. Pío XII, 36 \\ 31008 Pamplona
}




\section{INTRODUCCIÓN}

El rápido progreso en la tecnología médica ha favorecido la aparición de nuevas formas de tratar las enfermedades. Esto está ocurriendo en gran parte gracias al desarrollo de nuevas técnicas de la imagen y del diseño y fabricación de novedosos dispositivos pero también gracias a un progresivo cambio en la formación de los nuevos médicos. A esto se añade una permanente investigación, constante búsqueda de innovadoras alternativas terapéuticas con las que se pueden tratar mas, mejor o más rápidamente las enfermedades. Las técnicas a las que estamos haciendo referencia y denominadas "mínimamente invasivas", están por tanto basadas en "imagen, investigación y formación". La artroscopia, la laparoscopia, la endoscopia digestiva, urológica o bronquial son, entre otras muchas, sólidas y reconocidas técnicas y procedimientos terapéuticos que ahora están en primera línea, no ya como alternativa a las denominadas como "convencionales" sino como tratamiento de elección, o único, en muchos casos.

De los departamentos de radiología y del innovador espíritu de los radiólogos, nacieron, a partir de la década de los 60 , un gran abanico de procedimientos, ahora denominados "endovasculares" con los que, utilizando el soporte de la imagen radiológica y con un verdadero alarde de imaginación, se revolucionó el tratamiento de la patología vascular. La angioplastia con balón o con endoprótesis metálica, la aterectomía percutánea, la oclusión vascular o la trombolisis directa son el inicio de una larga lista de procedimientos cuyo desarrollo y perfeccionamiento, unido a un cambio en la formación clínica del médico, han dado contenido a una nueva especialidad médica llena de futuro y denominada por algunos como "radiología intervencionista".

En este breve capítulo se pretende exponer, a modo de conciso catálogo, algunos de los campos de aplicación de esta nueva especialidad. Especialidad entendida como disciplina médica en la que, en ocasiones, médicos diferentes de los radiólogos participación activa y exitosamente en su desarrollo e implantación en la práctica médica.

En concreto se presentan aspectos sobre las técnicas, los materiales y los resultados clínicos obtenidos en patología vascular, en patología hepática y en el control y tratamiento de las hemorragias. En razón de la brevedad del artículo, importantes (y centrales) campos del intervencionismo como son los "accesos venosos" o el manejo percutáneo de la patología biliar o la urológica son, no han sido tratados.

\section{PATOLOGÍA VASCULAR}

La enfermedad arterial oclusiva sintomática afecta al $5 \%$ de los varones mayores de 50 años. La claudicación intermitente, su manifestación más común, puede producir dolor de la extremidad tanto en la marcha como en reposo, teniendo como resultado una severa limitación de la movilidad. La cirugía, fundamentalmente el bypass, es un eficaz tratamiento para estos pacientes y cuya indicación mas clara será en aquellos que presenten isquemia crítica.

Muchos pacientes con claudicación, independientemente de la localización de la lesión vascular, podrán ser tratados con técnicas endovasculares. La más común, de primera línea, es la angioplastia con balón de cuyos resultados se hablará en los próximos apartados. El problema más común es la recidiva de la lesión estenótica (que oscila, según territorios, entre el $5 \%$ a los 3 años y el $30-60 \%$ a los $6-12$ meses). Esta reestenosis dependerá de muchos factores entre los que se incluyen la longitud de la lesión vascular que se ha tratado, si se trata de una oclusión o de una estenosis, o de su localización. El sustrato histológico de la reestenosis, basado en la remodelación vascular, es complejo y una de sus causas es la proliferación excesiva de células de músculo liso con formación de una gruesa neoíntima ${ }^{1}$.

El mayor conocimiento sobre la formación de la lesión vascular o de las causas por las que aparece la reestenosis ha permitido desarrollar nuevas estrategias terapéuticas basadas en eficaces protocolos de antiagregación y anticoagulación. Del 
mismo modo, la investigación en la búsqueda de nuevos tratamientos ha permitido desarrollar y disponer de dispositivos como las endoprótesis metálicas o los sistemas de liberación lenta y controlada de fármacos en la lesión, por citar dos ejemplos. Las endoprótesis metálicas ("stents") están fabricadas con diferentes materiales, preferentemente acero (aleación de hierro, cromo y níquel) y nitinol (aleación de níquel y titanio). El nitinol, de menor fuerza radial, presenta la ventaja de tener "memoria térmica" lo que le confiere una mayor plasticidad tanto durante la fabricación y montaje, como durante su liberación. Por tanto, dependiendo del material con que están fabricados y de su construcción (angulación de varillas, proporción metal / no metal, etc.), los diferentes stents tendrán múltiples peculiaridades pudiendo así diseñarse modelos específicos para aplicaciones clínicas concretas. Por ejemplo, algunos serán "autoexpandibles", es decir, que una vez liberados del catéter que los transportaba adoptan la morfología con la que fueron fabricados. Estos dispositivos son muy útiles para el tratamiento de patología vascular (arterial o venosa) en territorios de gran calibre (por ej. ilíacas) o en patología no vascular (p. ej. biliar). Otros stents, los "expandibles con balón", irán montados sobre un balón de angioplastia. Para su liberación bastará el inflado del balón y el stent se adaptará a la pared del vaso patológico. Estos dispositivos se liberan, por tanto, de manera muy precisa y, en general, se dice que son de aplicación recomendada en vasos de mediano calibre (por ej. renales) y pequeño calibre (p. ej. coronarias) ${ }^{2}$.

Finalmente decir que algunos stents pueden ir cubiertos por tejido, habitualmente Dacron o PTFE (politetrafluoretileno). Estos dispositivos se aplicarán preferentemente en patología vascular aneurismática o traumática. Otros estarán "cargados" en su superficie con diferentes sustancias (antiagregantes, antineoplásicos, inhibidores del crecimiento celular, etc.) con las que se pretenderá obtener un efecto terapéutico añadido al del propio stent con una disminución de la respuesta "reestenótica” de la pared vascular afecta ${ }^{3}$.

\section{Estenosis y oclusiones arteriales}

\section{Sector aorto-ilíaco}

Es en este sector donde los resultados obtenidos con las técnicas percutáneas son, con mucho, los mejores y los más duraderos y, por tanto, los más aceptados. La cirugía queda reservada para casos con obstrucciones largas, vasos muy tortuosos o lesiones múltiples y difusas. Las series más amplias demuestran que los resultados obtenidos con la angioplastia con balón serán: éxito técnico en un $92 \%$, permeabilidad primaria a los 2 años del $81 \%$ y a los 5 años del $72 \%$. La tasa de complicaciones será del $4,5 \%$, de las que un $2,7 \%$ serán "mayores" (que requieren cirugía). En casos de obstrucciones cortas o con mal resultado morfológico tras angioplastia o en lesiones vasculares complejas (por ej. placas excéntricas, calcificadas, ulceradas, etc.) se recomienda el uso de stents. Parece que no hay grandes diferencias en cuanto al resultado clínico a largo plazo, según sea el stent seleccionado, y la permeabilidad (asistida o secundaria) resultante podrá ser del 96 al año, 93 a los 2 años y 91\% a los 3-4 años (4) (Fig. 1).

\section{Sector femoropopliteo}

Las lesiones de este territorio, fundamentalmente causadas por patología arterioesclerosa, se manifestarán clínicamente como "agudas" (trombosis brusca asociada a una silente enfermedad de base) o "crónicas". Para el tratamiento de lesiones "agudas" se han descrito diferentes procedimientos percutáneos. Destaca el empleo de fármacos trombolíticos (urokinasa o rtPA) aplicados directamente a la zona de oclusión. Con ellos, y en casos adecuadamente seleccionados, el éxito inicial puede ser del $90 \%$. En determinadas ocasiones, por ejemplo en oclusiones embólicas, una eficaz alternativa (también más rápida y barata) en la extracción directa del trombo mediante el uso de gruesos catéteres (8 French) y aspirado manual. Con esta técnica, denominada "tromboembolectomía por aspiración percutánea", se han descrito éxitos terapéuticos del $90 \%$, siempre en casos bien seleccionados. Para lesiones "crónicas" se han descrito múltiples posi- 

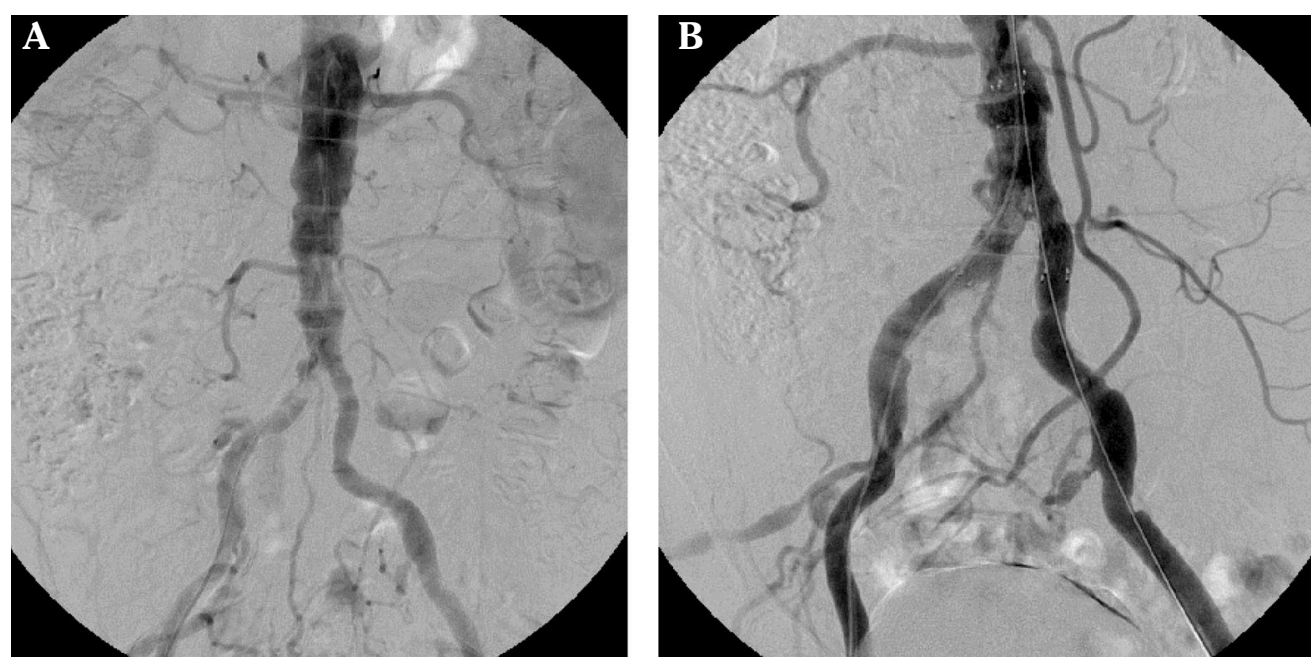

Figura 1. Paciente de 65 años con claudicación intermitente en extremidad inferior derecha. A)- Aortografía en la que se observa ateromatosis en la bifurcación aorto-ilíaca con estenosis significativa en el origen de la arteria ilíaca común derecha. B)- Dada la situación de la placa de ateroma (bifurcación aórtica) se colocan prótesis metálicas autoexpandibles en ambas ilíacas. El resultado morfológico y clínico fue satisfactorio.

bilidades terapéuticas, destacando entre ellas, y como método más sencillo y rápido, la angioplastia con balón, sin embargo los resultados a largo plazo no son del todo satisfactorios. Quizá la simplicidad de la técnica y la posibilidad de su repetición a lo largo del tiempo pueden justificar resultados tan bajos como un 50\% (en las mejores series) de permeabilidad a los 5 años. Se han utilizado stents, en un intento de mejorar la baja permeabilidad primaria obtenida con la angioplastia con balón, sin embargo los resultados finales no son mucho mejores. Otras técnicas como la recanalización con laser o el uso de stents cubiertos han aportado buenos resultados iniciales aunque, a largo plazo (5-7 años), ninguna técnica ha mejorado a la angioplastia con balón ${ }^{5}$.

\section{Arterias crurales}

En pacientes con afectación ateromatosa de las arterias del territorio infrapoplíteo el objetivo clínico fundamental que se pretende es "la salvación de la extremidad", es decir, evitar la amputación. Los resultados clínicos obtenidos, con una cui- dadosa técnica, pueden alcanzar una mejoría clínica del $80 \%$ a los 2 años $^{6}$.

\section{Arterias renales y viscerales}

A pesar de la gran cantidad de series publicadas y del elevado número de pacientes tratados con angioplastia de la arteria renal, sigue abierto el debate sobre qué pacientes deben de ser tratados con técnicas endovasculares o incluso, sobre qué pacientes deben ser tratados con técnicas de revascularización. Obtener éxito técnico o mejorar el flujo y la perfusión de un riñón no tiene porque verse obligatoriamente acompañado de una mejora en la situación clínica del paciente. Por todo esto, evaluar o extraer conclusiones sobre los resultados obtenidos con una técnica endovascular u otra puede ser difícil. Parece, sin embargo, que el empleo directo de stents ("balón expandibles") asegura una mejor permeabilidad, con una morbilidad menor, que usando exclusivamente la angioplastia con balón. Un reciente estudio presenta un éxito técnico del $88 \%$ con el manejo de stents y del $57 \%$ cuando se utiliza únicamente el balón de angioplas- 
tia. Las estenosis del origen de la arteria renal son debidas a la presencia de placas de ateroma en la pared aórtica y su tratamiento debe incluir el manejo de stents "de entrada" colocando su extremo proximal unos $2 \mathrm{~mm}$ dentro de la luz aórtica ${ }^{7}$.

Son escasas las series que aportan información sobre el seguimiento a largo plazo de pacientes con isquemia intestinal que han sido tratados con angioplastia, con o sin stent. La reoclusión a medio plazo del segmento tratado no implica la aparición de nuevos episodios sintomáticos, por tanto, los datos sobre permeabilidad son controvertidos. Parece, claro de todas formas que con stents se obtienen mejores resultados y que la permeabilidad, a los dos años, puede ser como en las arterias renales, del $75 \%$.

\section{Troncos supraaórticos}

Posiblemente el tratamiento de las estenosis carotídeas mediante técnicas endovasculares sea el procedimiento que genera mas interés y discusión en este momento. Tradicionalmente las indicaciones aceptadas, aunque también discutidas, para llevarlo a cabo incluían lo que se conoce como cuello "hostil", lesiones altas de difícil abordaje quirúrgico, lesiones inflamatorias o postrádicas y pacientes con comorbilidades o de alto riesgo quirúrgico. El cambio de milenio trajo consigo la aparición de nuevas prótesis especialmente diseñadas para su implantación en el territorio carotídeo, e incluso algunas con forma cónica. Pero quizás el avance tecnológico que más ha contribuido a reanimar el debate sea la comercialización de los denominados filtros o instrumentos de protección, que en teoría permiten realizar el procedimiento disminuyendo el riesgo de complicaciones embólicas. Actualmente, las indicaciones para la colocación de stents en carótida son la presencia de una estenosis carotídea, sintomática, superior al $70 \% \mathrm{y}$, en pacientes asintomáticos, en estenosis del $80 \%$ con oclusión carotídea contralateral.

Los detractores de este procedimiento argumentan la ausencia de estudios multicéntricos randomizados con suficiente seguimiento en el tiempo que demuestren la superioridad de esta técnica con respecto a la endarterectomía. Sin embargo, a día de hoy existe suficiente cúmulo de evidencia científica publicada en prestigiosas revistas que demuestran que este procedimiento es al menos equivalente a la endarterectomía tanto en resultados (éxito del $96 \%$ ) como en tasa de complicaciones en el postoperatorio inmediato (10\%), a los treinta días y a largo plazo ${ }^{9}$.

\section{Lesiones venosas}

Las técnicas endovasculares han demostrado su eficacia en una gran variedad de enfermedades y lesiones venosas. Por ejemplo, su uso ha permitido aumentar la permeabilidad de los accesos vasculares para pacientes que requieren hemodiálisis. En casos de obstrucciones largas del drenaje venoso se han aplicado diferentes técnicas con las que se ha obtenido, de forma rápida, una duradera permeabilidad. Son, entre otras, la "trombectomía percutánea”, aplicada casi exclusivamente para patología venosa y con especial interés en las fístulas para hemodiálisis. En muchas ocasiones, sin embargo, resulta igualmente eficaz la angioplastia con balón, no siempre asociada a la fibrinolisis con fármacos ${ }^{10}$.

En pacientes con "síndrome en esclavina" por obstrucción tumoral de vena cava superior, la implantación de una endoprótesis resulta un método rápido y, habitualmente, sencillo con el que se obtiene una inmediata y duradera mejoría sintomática. Mayor controversia hay, sin embargo, con el empleo de estos dispositivos en patología venosa benigna, tanto de cava superior como de cava inferior. Se han publicado series con una permeabilidad primaria del $60 \%$ al año de la implantación. Estos datos, excelentes en la mayoría de los casos "malignos" y aceptables en determinados paciente en hemodiálisis, no son tan favorables para pacientes con estenosis debidas a patología traumática, o compresión extrínseca (Síndrome de Paget-Schroetter p. ej.). Por tanto, en patología estenótica venosa de causa no tumoral, las implantaciones de stents deben de evaluarse de manera totalmente individualizada ${ }^{11}$ (Fig. 2). 

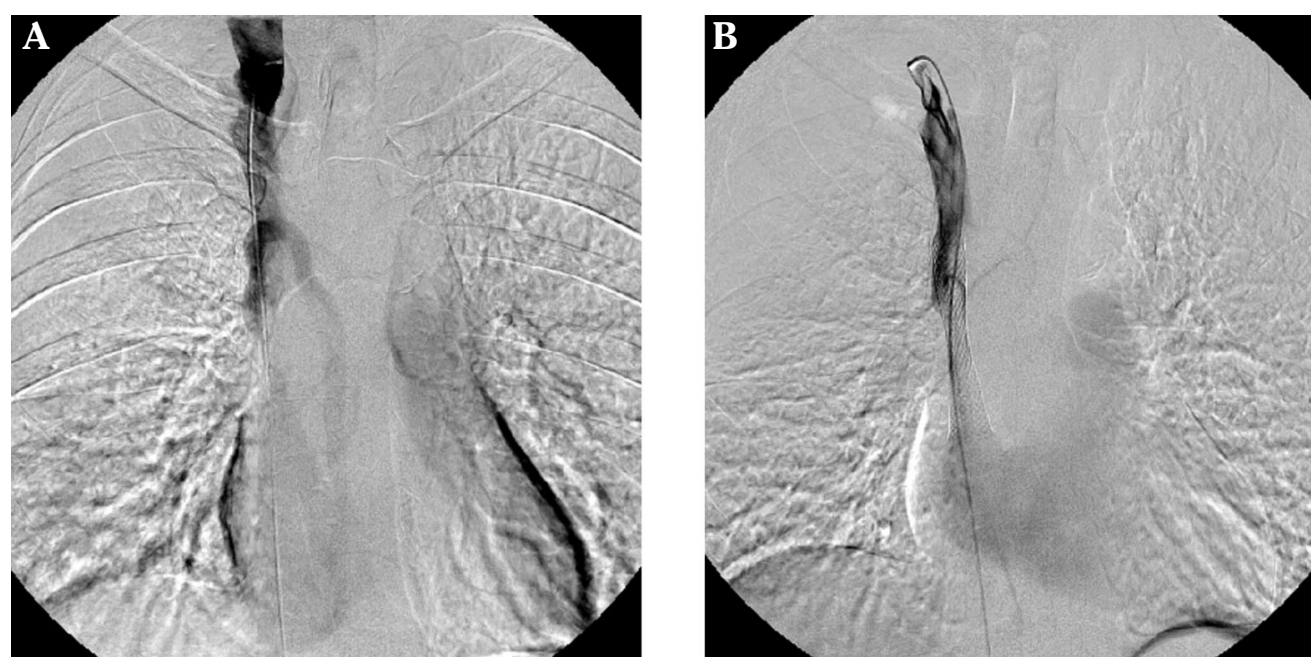

Figura 2. Paciente de 55 años con carcinoma inoperable de pulmón. Presenta edemas y enrojecimiento facial. A) Tras cateterización de vena femoral se realiza cavografía en la que se observa una estenosis crítica de cava superior con flujo invertido por la vena ázigos. B) Cavografía realizada tras la colocación de una endoprótesis. Se observa buen paso de contraste a cavidades cardíacas derechas sin relleno de la ven ázigos. La mejoría clínica del paciente fue inmediata.

\section{Aneurismas aórticos}

Aneurisma es una dilatación permanente y localizada de un vaso sanguíneo. Aunque no hay consenso, se define como aneurisma de aorta abdominal (AAA) a una dilatación con un calibre superior a $3 \mathrm{cms}$. Las dos terceras partes de todos los aneurismas arteriales son AAA. La incidencia de AAA ha aumentado llamativamente en los últimos años y ha pasado del $0,9 / 100.000$ habitantes/año en los 50 a 39/100.000 habitantes/año en los 90 .

La historia natural de un AAA es la expansión gradual y progresiva hasta que finalmente se produce la rotura, a menos que el paciente fallezca de otra causa no relacionada. El diámetro del aneurisma es el mejor predictor reconocido para rotura de AAA, de tal forma que la supervivencia actuarial, a cinco años, de aneurismas pequeños es del $48 \%$ cayendo al $6 \%$ en los mayores de $6 \mathrm{cms}$. Para un AAA con un diámetro transverso comprendido entre 5 y 7 cms el riesgo estimado actual de rotura es del 5 al $15 \%$. Por último, la presencia de trombo no implica una disminución de la posibilidad de rotura ya que, como sangre incompresible, transmite la presión que se ejerce sobre la pared.

La mejor prueba diagnóstica para detectar precozmente un AAA es la ecografía. El TC con reconstrucción 3D será el mejor método para conocer sus dimensiones y morfología, detectar signos de rotura, realizar diagnósticos diferenciales o complementarios y planificar, caso de precisarse, la mejor opción terapéutica.

Las series clásicas demuestran que el tratamiento quirúrgico del AAA es altamente eficaz con una mortalidad perioperatoria del $5 \%$. Se ha visto, sin embargo, que el aumento de la edad de la población y la precoz detección de los AAA (hasta diez veces mayor en el caso de los "pequeños") han favorecido la inclusión en series quirúrgicas de pacientes con patologías asociadas lo que, en algunos casos, ha elevado la morbi-mortalidad quirúrgica hasta en un $40 \%$ de los casos. Por este motivo, con el fin de evitar la laparotomía necesaria para la exposición y tratamiento quirúrgico del AAA, Parodi, en 1976, comenzó a desarrollar nuevos dispositivos, de implantación endoluminal, con los que proponía tratar a estos pacientes con menor mortalidad. 
Estos dispositivos estaban, y están, basados en la expansión radial y anclaje de endoprótesis metálicas autoexpandibles situadas en sus extremos y en unas estructuras tubulares, mas o menos reforzadas con mallas metálicas, cubiertas de tejido con el que se aisla al flujo arterial de la pared aneurismática. Estos dispositivos (endoprótesis) diseñados específicamente para cada paciente, y confeccionados gracias a los datos obtenidos con el TC, están construidos con diferentes "módulos" con lo que se facilita su implantación. Pueden ser "tubulares", cubriendo solo la aorta o la aorta y una ilíaca o "bifurcados", cubriendo la aorta y ambas ilíacas. Proximalmente no deben cubrir el origen de las arterias renales y se requiere un "cuello" de superficie aórtica en el que no haya ni aneurisma, ni trombo ni placa de ateroma para que la prótesis se fije y cierre la entrada proximal al AAA. Distalmente, dependiendo de la morfología de los vasos del paciente y de las peculiaridades del dispositivo seleccionado, se intentará evitar la oclusión del origen de las arterias ilíacas internas con el fin de evitar la isquemia del colon izquierdo o la temida claudicación glútea. Los dispositivos se colocarán tras la exposición quirúrgica de una o ambas arterias femorales comunes.

El éxito técnico de la colocación y sellado del AAA es superior al $97 \%$ y los fracasos terapéuticos vendrán por la aparición de las fugas ("endoleaks"). El tiempo quirúrgico y las necesidades de sangre o plasma son significativamente menores que con la técnica clásica. Las "reconversiones", definidas como la necesidad de cambiar la técnica endovascular por la abierta, son inferiores al $2 \%$. Los "endoleaks" se han clasificado en cuatro grupos:

Tipo 1. Los relacionados con los sitios de anclaje de las prótesis.

Tipo 2. No relacionadas con la prótesis y debidas al flujo retrógrado de los vasos que se originan en el AAA.

Tipo 3. Por defectos de la prótesis o por filtraciones entre los segmentos.

Tipo 4. Debidas a la porosidad del material protésico (Fig. 3).
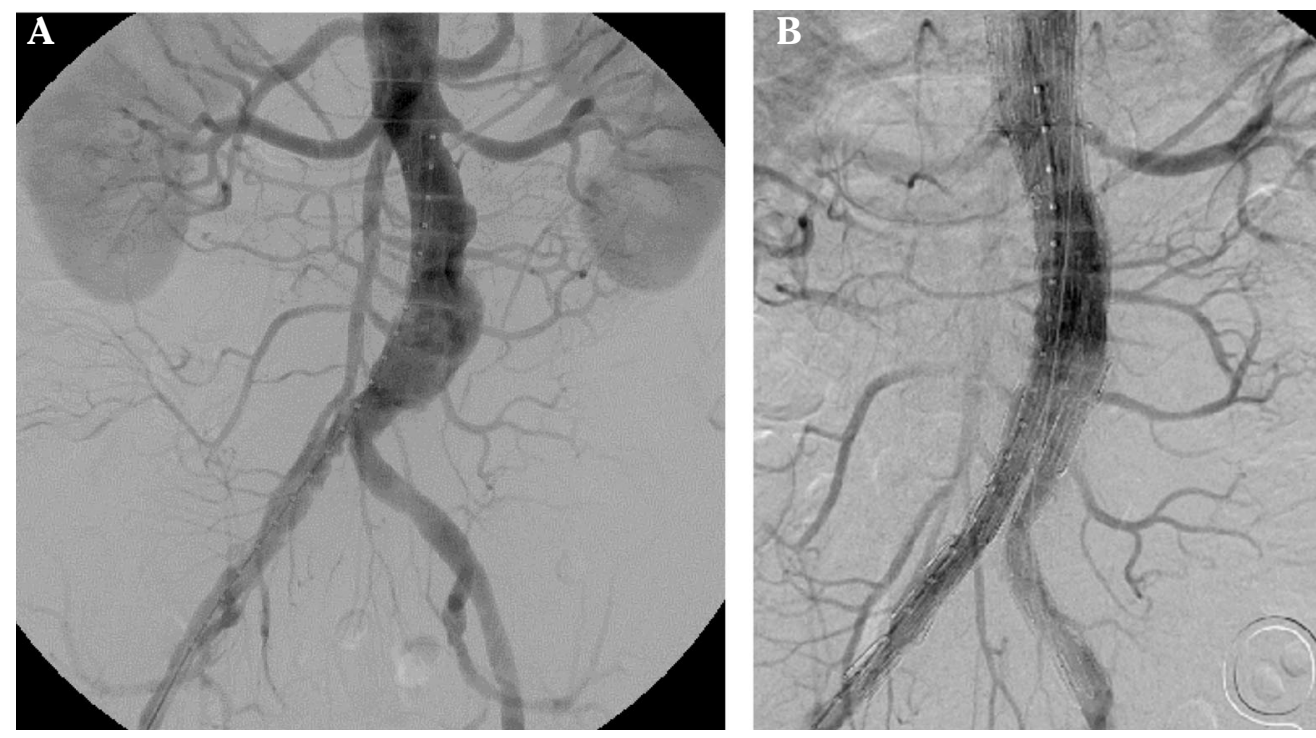

Figura 3. Paciente de 72 años con aneurisma de aorta abdominal. Por múltiples antecedentes clínicos, no era posible realizar su tratamiento por laparotomía. A) Aortografía en la que se observa una dilatación aneurismática de la aorta abdominal. El aneurisma también afectaba a la arteria ilíaca común derecha. B) Tras la colocación de una endoprótesis bifurcada, con "anclaje" suprarenal, se observa buen flujo por las arterias renales y exclusión del aneurisma. 
Aunque los resultados inmediatos y a medio plazo son muy satisfactorios, en cuanto a disminución del tamaño del AAA o rotura del mismo, permanecen abiertos muchos aspectos como el tamaño del AAA a partir del cual debe de ser tratado con estas técnicas o el comportamiento de las endoprótesis a largo plazo pues la lesión cambia en tamaño, angulación y morfología de forma que, teóricamente, puede haber acodamientos o fracturas del material ${ }^{12}$.

Los mismos principios en cuanto a material y abordajes se han seguido, con importante éxito terapéutico, en la aorta torácica. Se han publicado múltiples series en pacientes con aneurismas, disecciones con origen en esta localización o roturas traumáticas. Si embargo, por la brevedad el artículo, no se presentarán los resultados en esta localización.

\section{PATOLOGÍA HEPÁTICA}

El hígado presenta, desde el punto de vista vascular, una importante peculiaridad y es que presenta doble vascularización aferente. La arteria hepática, rama del tronco celíaco, y sujeta a una gran diversidad de variantes anatómicas, aporta al hígado el $30 \%$ del aflujo vascular y nutre de forma prácticamente exclusiva los tumores hepáticos, tanto primarios como metastásicos. Esto ocurre porque los tumores generan agentes pro-angiogénicos, con los que "se aseguran" un aporte arterial. La vena porta conduce el $70 \%$ restante de la vascularización hepática. Es un sistema "cerrado" formado por las venas mesentéricas y esplénicas y conectado con la vascularización sistémica por múltiples venas colaterales, fundamentalmente gastroesofágica, espleno-vascular y hemorroidales. El drenaje venoso hepático es único (venas suprahepáticas) y, al igual que las arterias, está sujeto a múltiples variantes anatómicas.

\section{Hipertensión portal}

Cualquier circunstancia que eleve el gradiente porto-sistémico (diferencia de presión entre el sistema portal y la presión venosa central) por encima de 10-15 mmHg tendrá como consecuencia el desa- rrollo, y su posible rotura, de las colaterales que conectan el territorio portal y las venas sistémicas. El planteamiento terapéutico de esta compleja situación se puede plantear básicamente, de tres formas diferentes: En pacientes cirróticos, tratar la causa como por ejemplo con un trasplante hepático, disminuir el gradiente portosistémico de manera farmacológica o con técnicas derivativas o asegurar la hemostasia del punto de sangrado mediante terapia endoscópica o percutánea (embolización, escleroterapia, etc.)

Las técnicas endovasculares han demostrado su utilidad y gran eficacia en los tres apartados mencionados previamente. Aunque no es nuestro deseo referirnos a "técnicas terapéuticas" sino a formas de tratar a pacientes con patología hepática, con el fin de ser concisos, a continuación se describen las diferentes posibilidades técnicas paliativas y curativas que la radiología intervencionista aporta para el tratamiento de los pacientes con hipertensión portal.

\section{Tratamiento de las enfermedades causantes de obstrucción en el drenaje venoso}

Fundamentalmente el síndrome de "Budd-Chiari" y la obstrucción de las venas hepáticas causadas, a veces, por membranas "congénitas" aunque, cada vez con más frecuencia, por complicaciones postquirúrgicas, sobre todo del trasplante hepático. Para estos casos, el acceso transparietohepático, dirigido con ecografía, y la angioplastia simple o con colocación de un stent, ofrece resultados duraderos y quizá curativos. La tasa de reestenosis en estos pacientes es muy baja (permeabilidad a los tres años del 90\%) y las complicaciones del procedimiento son escasas.

\section{Tratamiento de las enfermedades causantes de hipertensión portal por afectación parenquimatosa hepática}

Independientemente de si la lesión es "presinusoidal", "sinusoidal" o "postsinusoidal"; el tratamiento en estos casos será paliativo. Aunque hay otros, quizá el método terapéutico más conocido sea el TIPS 
(acrónimo de "Transjugular Intrahepatic Portosystemic Shunt"), Este singular procedimiento, basado en la conexión percutánea directa entre dos venas, consiste en la punción, transparenquimatosa, de la vena porta desde la vena hepática. Cuando se describió en 1969, se abrió un nuevo campo de investigación, completamente nuevo, que plantea la conexión directa de dos vasos sanguíneos. A lo largo de los años se fueron desarrollando diferentes aspectos técnicos y ha pasado a ser procedimiento bien instalado y reconocido en el tratamiento de pacientes con hipertensión portal. El éxito técnico supera el $90 \%$ y, en pacientes con hemorragia aguda que mantengan una buena función hepática (Child A o B), ofrece una supervivencia elevada (supera el 70\%) al mes de su realización (Fig. 4).

A pesar de asegurar la supervivencia a pacientes que han recidivado a otros tratamientos y de mejorar la situación clínica e muchos pacientes, el TIPS tiene dos "talones de Aquiles". En primer lugar la "encefalopatía crónica" (inferior al 13\%) y en segundo lugar la reestenosis. Las prótesis "no cubiertas", si bien son muy eficaces para crear un canal derivativo directo de la porta, tienden a ocluirse. Las razones son múltiples, entre ellas la "contaminación" intraductal de material biliar debido a la transección de canalículos biliares en el trayecto del TIPS. Es por esta razón por la que se han estudiado dos posibles formas de solucionar el problema de la mencionada reestenosis, en primer lugar está la creación de conexiones percutáneas portosistémicas totalmente extrahepáticas y en segundo lugar la utilización de prótesis cubiertas de material no permeable (PTFE). Con estos avances, la permeabilidad primaria de los TIPS ha cambiado radicalmente (de un $50 \%$ al año ha pasado al $90 \%$ ) de tal forma que ahora, en pacientes en los que los tratamientos farmacológicos y endoscópicos han fracasado, el TIPS es la mejor alternativa, la mejor técnica derivativa, para pacientes con problemas derivados de la hipertensión portal ${ }^{13}$.
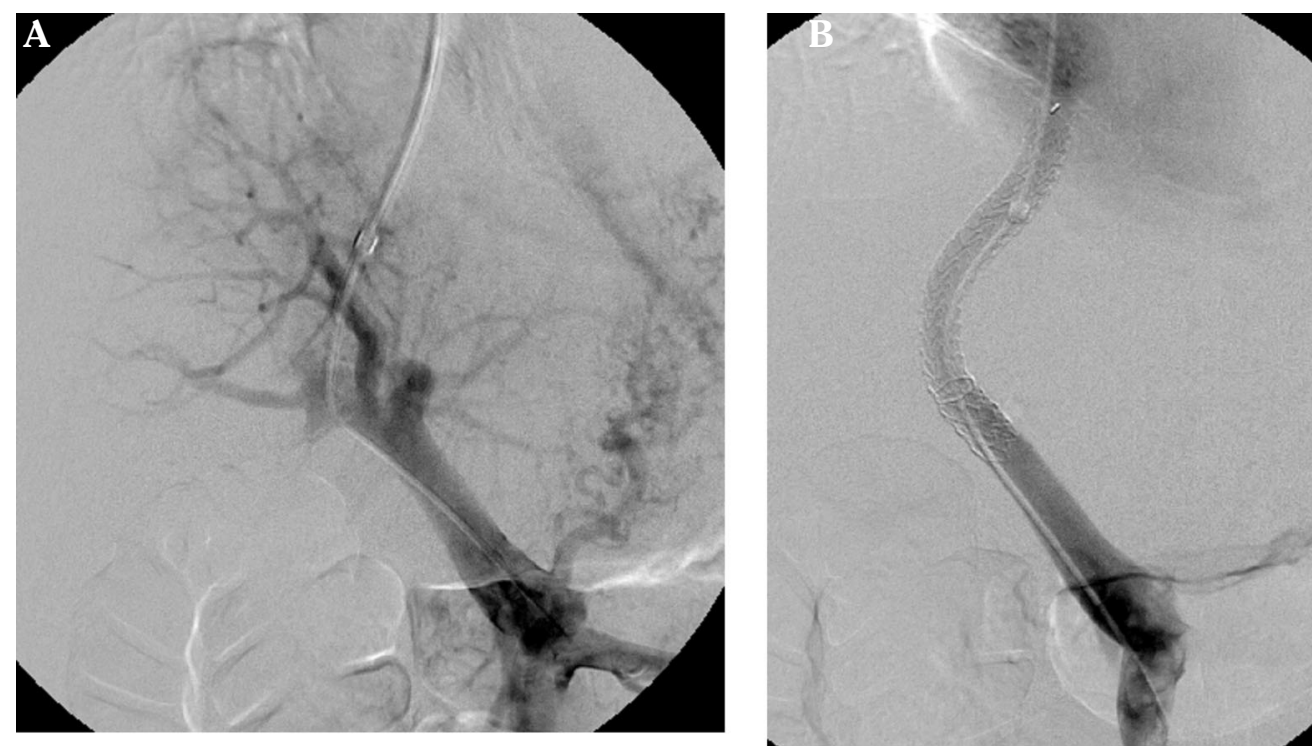

Figura 4. Paciente cirrótico de 52 años con hemorragias recidivantes por varices gástricas. A) Portografía realizada tras la punción transyugular de la vena porta derecha. Se observa relleno hepatofugal de varices dependientes de la vena coronaria estomáquica. B) TIPS realizado con una endoprótesis metálica cubierta. Se observa buen flujo portal y ausencia de relleno de las varices. 


\section{Lesiones de vena porta}

Las causas por las que puede generarse hipertensión portal por alteraciones en la vena porta, y sus ramas afluentes, son múltiples. Entre ellas se encuentran las estenosis u obstrucciones de causa inflamatoria (pancreatitis, pileflebitis, etc.) y las de causa quirúrgica (post trasplante hepático por ejemplo). En ausencia de patología hepática asociada, el tratamiento más adecuado será la realización de una angioplastia, preferentemente con colocación de stent, realizada por acceso portal directo transparietohepática. La permeabilidad a medio plazo (3-5 años), si bien los datos son escasos, es elevada.

\section{PATOLOGÍA TUMORAL}

En la práctica, y muy probablemente porque todavía las modalidades terapéuticas son escasas y limitadas, se tiende a unificar el tratamiento de diferentes entidades que, ubicadas en la misma víscera (el hígado), tienen etiologías, patogenias y evoluciones tan diferentes como los hepatocarcinomas y las metástasis de tumores tan diferentes como pueden ser los neuroendocrinos o colorrectales. Utilizando los mismos argumentos que los expuestos en el apartado "hipertensión portal", se presentan a continuación las "modalidades terapéuticas" más utilizadas en estos momentos para la patología tumoral hepática.

\section{Quimioterapia intraarterial}

Un elevado porcentaje de los pacientes con carcinoma del territorio colorrectal presentan metástasis hepáticas (15-20\% en el momento del diagnóstico y un 20\% durante la evolución clínica posterior). En estos casos, la supervivencia media será de solo 9 meses. Con la mejora de las técnicas quirúrgicas y gracias a una mayor precisión en la estadificación tumoral, utilizando técnicas radiológicas, la resección de las lesiones hepáticas ofrece una supervivencia a 5 años que supera el $40 \%$. El problema es que solo un $20 \%$ de los pacientes con metástasis hepáticas pueden ser tratados de esta manera. Con el fin de "rescatar" pacientes y poder incluirlos en las listas quirúrgicas, se han descrito técnicas como la "embolización portal prequirúrgi- ca" (de la que no se va a tratar en esta revisión) con las que se obtienen muy buenos resultados. O también la combinación de cirugía y aplicación percutánea de radiofrecuencia a determinadas lesiones.

Pero, como se ha dicho anteriormente, hay todavía un elevado número de pacientes a los que únicamente se pueden ofrecer tratamientos paliativos, bien con la intención de "rescatarlos" para la cirugía o bien para aumentar los ya de por sí cortos periodos de supervivencia. Los resultados obtenidos, en las series más favorables, utilizando quimioterapia sistémica son de "respuestas" del 20-50\% con escasa mejora de la supervivencia. Por ello, desde los 60 se pensó que incrementando localmente la dosis del fármaco en la zona tumoral ("infusión intra-arterial de quimioterapia”), mediante el empleo de técnicas y dispositivos que así lo permitan, se elevarían las "respuestas" y, quizá también, la supervivencia. Según series recientes, los resultados obtenidos con el manejo intraarterial de fármacos son esperanzadores dado que haty estabilización /respuesta de la enfermedad en mas de un $70 \%$ de los casos con cifras de supervivencia que alcanzan el $25 \%$ a los dos años.

El fármaco puede administrarse con catéteres implantados quirúrgicamente en la arteria hepática sin embargo esta técnica apenas se utiliza actualmente dado el elevado número de complicaciones trombóticas que aparecen durante el seguimiento y la necesidad de practicar una laparotomía para su implantación. Por tanto la alternativa percutánea pasa a ser una primera opción pues los dispositivos ("reservorios y catéteres") pueden implantarse por vía endovascular y con punción arterial a distancia (femoral o axilar). Son muchas las técnicas descritas, así como diversos los materiales empleados, pero en todos los casos el mayor problema, y por tanto riesgo, es la movilización del extremo distal ("hepático") del catéter. Por tanto, cuando se coloque un reservorio intravascular será necesario ocluir (embolizar) todos los vasos que, desde la arteria hepática, aporten flujo a territorios no hepáticos (arterias gastroduodenal y gástrica derecha, por ejemplo) y también será preciso aplicar dispositivos que fijen el 
extremo distal del catéter, con los que se evite una inadecuada perfusión de fármacos a territorios no deseados. Con todo, la colocación percutánea de estos dispositivos ha aportado un gran beneficio a los pacientes pues permiten realizar repetidas aplicaciones de tratamientos sin necesidad de hospitalización ${ }^{14}$.

\section{Embolización}

Embolizar consiste, básicamente, en introducir en un vaso, de forma voluntaria, partículas o agentes con los que se pretende obtener un beneficio terapéutico. "Embolizar" en patología tumoral hepática tiene claras ventajas pues los tumores se nutren solo por arteria hepática por tanto, teóricamente, las partículas introducidas por vía arterial actuarán solo sobre el tejido tumoral ya que el "sano" recibirá su aporte vascular por vía portal. Con la embolización se pretende producir isquemia y, por tanto, necrosis celular. Sin embargo, la isquemia no solo produce necrosis sino también la liberación de factores neoangiogénicos con los que el tumor se pretende asegurar un nuevo aporte nutritivo. Es ésta, en gran parte, la causa de las recidivas tras embolización y, de aquí, la razón por la que la técnica, que además no está exenta de morbilidad ("síndrome post-embolización", p. ej.), tenga sus detractores. Tal es así que, en estos momentos, la "embolización" no se encuentra entre las primeras opciones "curativas" de un paciente con hepatocarcinoma ${ }^{15}$.

Por todo lo expuesto más arriba se ha visto la necesidad de mejorar y actualizar muchas de las técnicas y materiales de embolización. Las principales líneas podrían resumirse en dos. En primer lugar está el perfeccionamiento de los materiales, tanto con el desarrollo de nuevos catéteres "coaxiales" de bajo calibre (2,5 French, aproximadamente $1 \mathrm{~mm}$ ) con los que se puede acceder a finos vasos tumorales como con la investigación sobre nuevas partículas embolizantes. Resulta, por ejemplo, sorprendente conocer que importantes y conocidas series sobre pacientes tratados con esta técnica han utilizado agentes como la "esponja de fibrina", material de grueso calibre, de oclusión imprevisible y de efecto muy poco duradero. Al menos teóricamente, parece mucho más recomendable utilizar partículas esféricas, de tamaño y acción previsible, fabricadas con material inerte y de efecto duradero. En segundo lugar esta el hecho de perseguir, aprovechando la oclusión vascular y la isquemia que ofrecen la "embolización" un efecto añadido que asegure la necrosis tumoral. Hace 20 años apareció en Japón el concepto de "quimioembolización" que se basaba en el hecho de añadir un fármaco, mezclado con Lipiodol (contraste yodado liposoluble afín al tejido tumoral), a las partículas embolizantes, por aquel entonces, no esféricas. Se argumentaba que la isquemia favorecía la permeabilidad de la membrana celular y, por tanto, la entrada del fármaco al tumor. Los resultados iniciales fueron satisfactorios $\mathrm{y}$, en tumores únicos de hasta $5 \mathrm{cms}$, las cifras de supervivencia eran similares a las de la resección quirúrgica. Se desarrolló, entonces, un concepto nuevo que consistía en que las partículas podían ser "transportadoras" de diversos agentes. Estos pueden encontrarse fuera de las partículas de tal forma que el enlentecimiento del flujo (obtenido con la embolización) aumenta la exposición del agente. Este agente no tiene porqué ser obligatoriamente un fármaco antineoplásico (adriamicina, p. ej.) habiéndose presentado series con ácido acético o contraste yodado hiperosmolar. Los agentes también pueden transportarse en la superficie de las partículas (Itrio 90, p. ej.) o en su interior (adriamicina, p. ej.). Esto se ha obtenido gracias al desarrollo de nuevas partículas esféricas realizadas con materiales inertes, que producen escasa reacción inflamatoria y que permanecen largos tiempo sin deteriorarse o desestabilizarse. Se han presentado datos muy iniciales empleando partículas que llevan en su interior adriamicina; se sabe que la presencia de este fármaco es muy escasa en la circulación sistémica, asumiéndose que permanece en el hígado y próximo al tumor. Las respuestas clínicas que se han reportado son satisfactorias. Cabe la duda de saber si es la adriamicina el fármaco que se debe de usar pues no hay datos comparativos con otros como el 
cisplatino o la mitomicina, incluso si cualquiera de los tres es realmente eficaz en el hepatocarcinoma. Otra posibilidad totalmente diferente es añadir partículas, tipo Itrio 90, que, emitiendo radiación beta, produzcan una auténtica radioterapia intravascular aplicada directa y exclusivamente al área tumoral. En este caso, las partículas que se han diseñado son de 35 micras (quizá excesivamente pequeñas) y están fabricadas con resina. La selección de los pacientes tratados con esta novedosa técnica incluye a aquellos que, manteniendo buena función hepática, han recidivado o no son candidatos a otro tratamiento. Se han obtenido excelentes respuestas con un apreciable aumento en la supervivencia.

\section{TRATAMIENTO ENDOVASCULAR DE LAS HEMORRAGIAS}

El manejo terapéutico de una hemorragia digestiva se debe de enfocar de manera multidisciplinar y, a su vez, de modo personalizado. Dependiendo de los antecedentes y la situación clínica, por un lado, y del grado y localización de la hemorragia por otro, se utilizarán unos u otros métodos terapéuticos. Con independencia de si es la cirugía o la endoscopia o el tratamiento endovascular el método seleccionado, los objetivos terapéuticos deberán ser siempre los mismos: obtener hemostasia y tratar la lesión de base.

A continuación se resumen los resultados obtenidos con las técnicas endovasculares, habitualmente la embolización en el manejo de la hemorragia según diferentes localizaciones.

\section{Hemoptisis}

La hemoptisis masiva es una situación clínica grave. Se define como tal a la presencia de una hemorragia de origen pulmonar que sea mayor de $250 \mathrm{cc}$ al día o que cause insuficiencia respiratoria grave. La causa es muy variable pero, en nuestro medio, lo más común es que se trate de bronquiectasias de origen tuberculoso. La lesión se nutre por vasos de origen bronquial siendo muy infrecuente (malformaciones vasculares, p. ej.) que tengan como origen a ramas de la arteria pulmonar. Por tanto, en un paciente con hemoptisis severa o recidivante que no ceda con los tratamientos habituales un posible tratamiento será la embolización de la rama sangrante identificada tras la realización de arteriografías selectivas.

Las arterias bronquiales presentan un origen muy variable. Habitualmente son ramas de la aorta torácica descendente y están sujetas a un gran número de variantes anatómicas, pudiendo originarse también en la arteria subclavia, conjuntamente con las frénicas o, incluso, desde la arteria vertebral. Además, de la bronquial derecha pueden originarse arterias medulares. Por todo ello el estudio arterial de una hemoptisis debe de realizarse con especial atención así como con un buen conocimiento del material que se utiliza ya que las complicaciones, si bien bajas (inferiores al 5\%), pueden ser importantes.

Los materiales embolizantes deberán tener un tamaño adecuado ya que si alcanzan vasos excesivamente distales producirán necrosis bronquial y si ocluye la arteria en un lugar excesivamente proximal se facilitará la formación de colaterales que repermeabilizarán, en pocos días, el punto de sangrado apareciendo nuevamente la hemoptisis. No se deberá por tanto de utilizar líquidos (alcohol, p. ej.) ni partículas esféricas inferiores a 300 micras, tampoco oclusores tipo "coils" de manera exclusiva. Dada la peculiar morfología de las arterias bronquiales así como el particular requerimiento de una meticulosa técnica de embolización, los procedimientos se realizan, en la mayoría de los casos, utilizando finos catéteres coaxiales lográndose así eficaces embolizaciones superselectivas.

Los resultados obtenidos con la embolización son buenos. El éxito técnico puede ser superior al 95\% (algo peor en aspergilosis o neoplasias) y la recidiva a los cuatro años será inferior al $20 \%{ }^{16}$.

\section{Hemorragias de origen hepático}

Una lesión arterial hepática puede manifestarse como un sangrado libre intraperitoneal, como un hematoma intrahepático o subcapsular o como una hemobilia cuya manifestación última sería una hemorragia digestiva en principio difícil de dis- 
tinguir clínicamente de otras hemorragias de causa intestinal. Si la lesión es de la arteria hepática común o propia, en su trayecto extravisceral, la hemorragia será habitualmente masiva y de difícil control. La causa será un pseudoaneurisma, relacionado con cirugía previa (por ej. transplante hepático) con o sin infección asociada. El tratamiento consistirá en la embolización del punto de sangrado. Con el fin de evitar problemas isquémicos tras la embolización de la arteria hepática común es muy recomendable mantener permeable la arteria gastroduodenal para asegurar la perfusión arterial hepática. Si la embolización no es posible o, por razones anatómicas, no es conveniente se ha descrito, si bien en casos aislados, la reparación de la lesión vascular mediante la colocación de endoprótesis cubiertas con las que se sellará el punto de hemorragia manteniendo, a su vez, el flujo arterial.

Las lesiones vasculares intrahepáticas causantes de hemorragia digestiva (en muchos casos) se pueden dividir, morfologicamente, en cuatro tipos diferentes:

a. Lesiones de pequeños vasos terminales. Habitualmente causadas por agujas de biopsia hepática o catéteres biliares pueden producir grandes hemorragias.

b. Laceraciones vasculares. Casi siempre causadas por la introducción de catéteres de drenaje o por heridas o traumatismos lacerantes. Es bien conocido que, en estos casos, la lesión debe ser tratada tanto en su lecho distal como proximal. La arteria debe de ser embolizada (coils) inicialmente en la parte distal a la lesión y la razón es evitar que, por colaterales intrahepáticas, se produzca "reperfusión" distal del punto de sangrado. Finalmente se embolizará la arteria proximal a la lesión.

c. Lesiones vasculares con shunt aterioportal. El shunt arterioportal de origen traumático/yatrogénico es a veces la única manifestación angiográfica de una hemobilia no masiva e intermitente y ocluyendo la conexión arterio-portal se sellará también el punto de hemorragia. d. Laceración vascular con formación de pseudoaneurisma. El ejemplo más frecuente es el de una lesión de la arteria hepática derecha o de la hepática propia tras cirugía hepática o, sobre todo, tras colecistectomía laparoscópica. En estos pseudoaneurismas de gran tamaño, al igual que en las laceraciones vasculares, el tratamiento percutáneo más adecuado consistirá en la oclusión distal y proximal de las arterias con el fin de excluir la lesión arterial preservando el flujo distal. En ocasiones este procedimiento no es, técnicamente, posible o es ineficaz de tal modo que se deberá recurrir a otras posibilidades como, por ejemplo, el tratamiento directo de la lesión mediante su punción directa y trombosis utilizando coils, esponja de fibrina o, quizá lo mejor, trombina ${ }^{17}$ (Fig. 5).

\section{Hemorragias del tubo digestivo alto}

Las hemorragias digestivas altas son aquellas que tienen su origen por encima del ángulo de Treitz. Comentadas ya las que conectan con el tubo digestivo por papila de Vater (hígado) y excluyendo las que tienen origen venoso, relacionadas con hipertensión portal y sangrado por varices, el presente apartado se centrará en las hemorragias que se originan en el estómago y el duodeno.

\section{Estómago}

La vascularización gástrica se caracteriza por originarse de múltiples pedículos. Como mínimo están involucradas las arterias gástrica izquierda (o coronaria estomáquica), gástrica derecha (con origen en arterias hepáticas), gastroduodenal (con su rama gastroepiploica derecha) y esplénica (de las que nacen las gástricas cortas y la gastroepiploica izquierda). En casos de hemorragia digestiva a partir de un único punto de sangrado (por ej. ulcus de stress) una embolización selectiva, utilizando segmentos de esponja de fibrina, es altamente eficaz y la necrosis debida a isquemia post-embolización es rara.

Debido a la mencionada abundancia de colaterales, se ha descrito para hemorragias gástricas difusas no controlables 

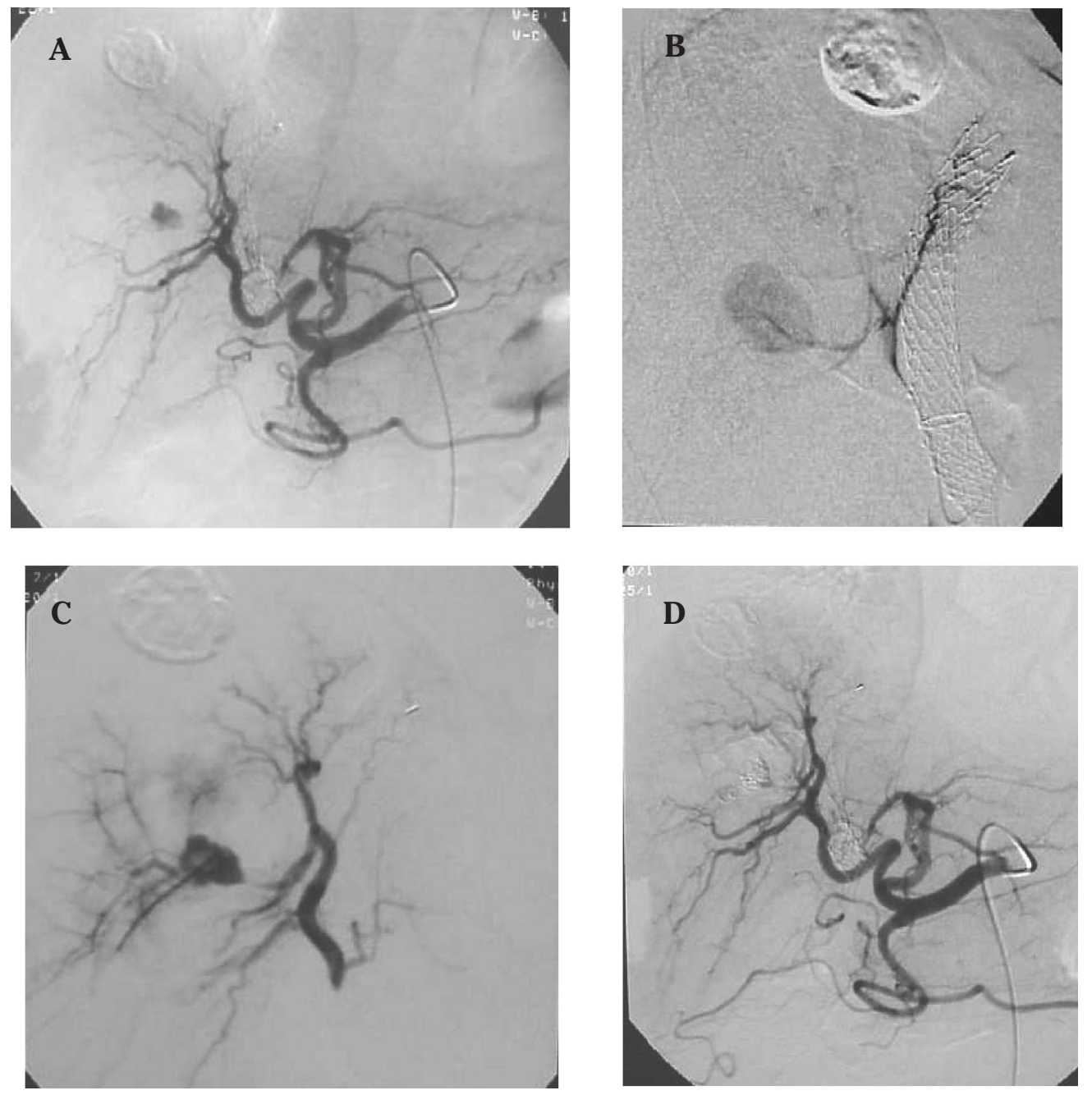

Figura 5. Paciente cirrótico de 70 años con antecedentes de hemorragias digestivas que requirieron la implantación de un TIPS. Presenta derrame pleural. Durante el intento de toracocentesis se punciona accidentalmente el hígado, el paciente presenta hemorragia digestiva. A) Arteriografía hepática en la que se observa un pseudoaneurisma intrahepático. B) Cateterización selectiva de una pequeña rama arterial que nutre parénquima hepático sano y vasculariza la arteria sangrante. Debido a la presencia de un TIPS permeable, que "roba" el flujo portal, se decide no embolizar con el fin de evitar la necrosis de parénquima sano. C) Arteriografía realizada durante la colocación percutánea de un catéter en el interior de la lesión. D) Arteriografía realizada tras la oclusión exclusiva de la lesión utilizando esponja de fibrina y coils.

con otras técnicas, la embolización no selectiva, también con segmentos de esponja de fibrina, de dos pedículos "principales", por ejemplo la gástrica izquierda y la gastro-epiplóica. Mediante esta técnica, el fin terapéutico que se busca es disminuir temporalmente el aporte sanguíneo para favorecer la hemostasia. Este procedimiento no se podrá realizar, evidentemente, en pacientes que hayan sufrido cirugía gástrica previa pues la víscera habrá perdido, en algunas zonas, su natural entramado de colaterales. 


\section{Duodeno}

La característica fundamental de la vascularización del duodeno es su doble aporte desde arteria hepática y desde arteria mesentérica superior. La vascularización duodenal, mediante la conexión de las arcadas pancreato-duodenales que se originan en las mencionadas arterias, funciona como un verdadero puente de conexión de alto flujo, entre ambas. Por tanto una hemorragia con origen en una lesión vascular (causada, por ejemplo, por un ulcus dudenal) deberá ser tratada atendiendo a este doble aporte. De tal forma que si a la lesión se accede por cateterización de la arteria gastroduodenal a través de la arteria hepática, el extremo del catéter o microcatéter se deberá de colocar distal a la lesión para, con coils, producir un sellado distal con el que se evite la entrada de sangre desde otro vaso ("reperfusión"). Una vez sellada la arteria distal, para tratar el punto hemorrágico se pueden emplear coils, segmentos de espongostan, partículas de grueso tamaño (300-500 m) o pegamentos $^{18}$.

\section{Hemorragias de tubo digestivo bajo}

Tal como se ha dicho anteriormente, se agrupan en este apartado a todas las hemorragias con origen distal al ángulo de Treitz. Las causas que lo producen son muy variadas, habrá territorios como el colon en los que los divertículos, tumores o malformaciones vasculares pueden ser detectados y tratados por técnicas endoscópicas, quedando para la cirugía o la embolización endovascular, un grupo reducido de pacientes. Por contra el intestino delgado es un territorio inaccesible a la endoscopia de tal forma que otras técnicas diagnósticas (como la angiografía) y terapéuticas (como la embolización o la cirugía) adquieren una relevancia mayor.

Desde comienzos de los años 80, se desarrollaron múltiples técnicas y materiales para favorecer la hemostasia de un vaso intestinal sangrante mediante la embolización del mismo. Sin embargo se observó que había complicaciones, fundamentalmente isquemia y resangrado, debidas a técnica inadecuada y mal uso del agente embolizante. Por este motivo se comenzaron a desarrollar otros métodos, como la infusión local de vasoconstrictores (por ej. Vasopresina). Este tratamiento requería cuidados especiales de hospitalización y no estaba exento de complicaciones cardíacas (isquemia miocárdica) o intestinales (por vasoconstricción excesiva). Aunque variaba según el territorio de origen del sangrado, pues era más eficaz en hemorragias de colon que de delgado, se describieron recidivas hemorrágicas en hasta un $50 \%$ de los casos. Debido a estos claros inconvenientes, se prestó nuevamente atención al desarrollo de técnicas de embolización. Nuevos materiales, como microcatéteres, partículas esféricas de embolización o microcoils fueron apareciendo en el mercado haciendo que la embolización fuese mucho más precisa y segura. Por tanto, a día de hoy, la embolización es una eficaz alternativa a la endoscopia y la cirugía, pudiendo recomendarse, desde el punto de vista técnico, para sangrados con origen en "cualquier lesión". Actualmente se acepta, como indicación de embolización, aquellos sangrados intestinales refractarios al tratamiento endoscópico o en aquellos en los que la endoscopia no sea factible, por ejemplo, en intestino delgado o en pacientes recientemente tratados con cirugía abdominal.

Cuando se va a realizar una embolización de una hemorragia digestiva baja, al igual que en otros tumores, se debe prestar atención no sólo a la obtención de hemostasia (de por si importante) sino al tratamiento específico de la lesión subyacente. No es lo mismo embolizar un tumor submucoso que una angiodisplasia o un aneurisma roto. Pero en general, para la gran mayoría de los casos, lo recomendable será realizar la oclusión vascular en un punto lo suficientemente distal, con lo que se evite isquemia de grandes tramos de intestino, y lo suficientemente proximal como para evitar isquemias de un segmento de pared intestinal. Este punto es el "vasa recta", arteria previa a los vasos murales y diferente en morfología y características, dependiendo de si es yeyuno, íleon o colon. En yeyuno son escasos y largos $(2-3 \mathrm{~cm})$ relativamente gruesos, en íleon son más abundantes, bien conectados por colaterales pero también más 
finos y en colon son escasos y nacen de la arteria marginal. La embolización de los "vasa recta" evita la isquemia mural, favorece la hemostasia, por disminución temporal del agente sanguíneo y no evita la revascularización por colaterales del territorio embolizado. Los materiales que se deben utilizar son microcoils o partículas como polivinil alcohol "no esférico" de 500-70 m o "esférico" de 700-900 m. Los resultados, atendiendo al éxito local (oclusión de la lesión) y clínico (detención del sangrado o aparición de complicaciones) variarán según la época de la publicación. Tales resultados se medirán atendiendo a la aparición de infarto o a la distensión del sangrado y podrán oscilar entre 0 y $20 \%$ para el primero y 0 y $42 \%$ para el segundo ${ }^{19}$.

\section{Hemorragias de origen traumático \\ Traumatismos pélvicos}

La mayoría de los pacientes con traumatismos pélvicos mejoran con las medidas terapéuticas oportunas y su situación hemodinámica es estable. Sin embargo, un $60 \%$ de los pacientes hemodinamicamente inestables fallecen por problemas derivados de una hemorragia masiva e incontrolada. Es por ello que el control local del teórico punto de sangrado adquiere gran importancia. La pelvis es un territorio anatómicamente complejo, con múltiples planos musculares y con vasos de grueso calibre. Tanto las arterias ilíacas (comunes, externas o internas) o sus ramas principales (glúteas, obturatriz, etc.) pueden sangrar masivamente cuando están rotas o laceradas. Esto ocurre en un 2-7\% de los traumatismos pélvicos y su manejo debe, por tanto, ser rápido. Tras la identificación del punto de sangrado, se procederá a su oclusión utilizando coils y el éxito técnico se acerca, según diferentes series, al 100\%. Cuando la lesión afecta a un vaso ilíaco (común, p. ej.) pueden colocarse stents recubiertos con PTFE, con ellos, si bien la información a largo plazo es todavía escasa, se aseguran tanto la hemostasia como la permeabilidad vascular.

\section{Abdomen y retroperitoneo}

En apartados precedentes se ha tratado el manejo endovascular de las hemo- rragias de origen hepático y gastrointestinal. Las lesiones traumáticas hepáticas, y esplénicas, deberán ser evaluadas con CT y el tratamiento de los posibles pseudoaneurismas, conexiones arterio-biliares, arterio-portales, será similar al descrito anteriormente.

La mitad de los traumatismos que causan problemas vasculares en el riñón son de causa yatrogénica (tras biopsias o nefrostomías). El manejo endovascular es habitualmente muy eficaz pues la identificación del vaso sangrante es sencilla y su oclusión altamente efectiva. Los vasos renales intraparenquimatosos se encuentran muy escasamente conectados entre si de tal forma que, por una parte, la oclusión del vaso producirá inmediata hemostasia pero también infarto del parénquima devascularizado. Por tanto, las embolizaciones deberán ser superselectivas y con microcoils o partículas esféricas de 500700 micras.

\section{Extremidades}

Las hemorragias debidas a traumatismos de extremidades pueden aparecer tanto de forma inmediata como días después de la lesión. Se presentarán en forma de pseudoaneurismas y/o roturas vasculares con o sin trombosis distal asociada. El tratamiento será, como mínimo, similar al que se realiza de forma quirúrgica. Los principios terapéuticos serán los mismos. Si la arteria dañada presenta abundantes colaterales distales se realizará oclusión distal (con coils), tratamiento de la lesión vascular (habitualmente con segmentos de esponja de fibrina) y oclusión proximal también con coils. Si la lesión es en un vaso de alto flujo, como ya se ha comentado anteriormente se recomendará la colocación de stents recubiertos. Si el vaso es "distal" (terminal) se embolizará con coils. Los resultados, siguiendo una técnica correcta, son satisfactorios ${ }^{20}$.

\section{Hemorragias de origen ginecológico}

Con una eficacia bien conocida desde hace ya varias décadas, la embolización es una alternativa terapéutica altamente útil en el manejo de los sangrados con origen gineco-obstétrico. Las indicaciones clási- 
cas y bien conocidas son las masivas hemorragias post-parto, las que tiene por origen un tumor o aquellas que surgen en el post-operatorio de una cirugía pélvica. El éxito vendrá relacionado con la identificación angiográfica del punto de sangrado y con la selección de un material adecuado con el que se consigue ocluir exclusivamente el vaso problemático. Conocidos estos hechos, hace casi 20 años se propuso utilizar la embolización dentro de la oferta terapéutica en pacientes con miomas uterinos.

Los miomas uterinos, formados por células de musculatura lisa, fibroblastos y colágeno, están presentes en un 25\% -50\% de las mujeres de entre 30 y 40 años. Si bien algunos son asintomáticos otros se manifestarán clinicamente con dolor pélvico, menorragia o polaquiuria por compresión vesical. La detección y estudio de inicio se realizará con ecografía y con la RM se obtendrá precisa información sobre el número exacto de lesiones, su ubicación en las distintas capas uterinas así como un buen seguimiento del comportamiento de las lesiones en caso de ser tratadas con técnicas no resectivas.

El tratamiento seleccionado dependerá de factores como la edad de la paciente, la gravedad de la sintomatología y el número y localización de los miomas. Con el tratamiento médico (agonistas de $\mathrm{GnRH}$ ) se conseguirá alivio sintomático pero no la desaparición de la lesión. El tratamiento quirúrgico, con el que se pretenderá resecar toda/s la/s lesion/es, podrá ser "miomectomía" (mediante laparotomía, laparoscopia o histeroscopia) o histerectomía (abdominal, vaginal o supracervical). Con ambas modadlidades se obtiene un elevado éxito técnico con un índice variable de complicaciones, fundamentalmente "fiebre" (5-10\%), "infección de la herida" ( $<5 \%)$ o "hemorragia" (2-5\%).

Técnicamente, la embolización de miomas uterinos consiste en cateterizar selectivamente ambas arterias uterinas y ocluirlas utilizando partículas (300-500 micras) no reabsorvibles. Morfológicamente la mejor indicación serán los miomas de localización "intramural" y, quizá también, los subserosos y los submucosos no pedunculados. No está indicada en el tratamiento de la infertilidad y tiene las mismas contraindicaciones que cualquier arteriografía.

El éxito inicial, definido como la oclusión de los vasos del interior del mioma con las partículas embolizantes, es del $98 \%$ siendo las causas mas comunes del fracaso, la embolización unilateral, el vasospasmo por la inadecuada manipulación de los catéteres o la presencia de vasos aferentes diferentes de las arterias uterinas (p. ej. las ováricas). La "complicación" inmediata mas frecuente es el dolor, en algunos protocolos se incluye la administración de anestesia epidural durante y después del procedimiento. Algunas pacientes presentan, además del dolor, el denominado "síndrome postembolización" con nauseas, vómitos y fiebre.

En un porcentaje superior al $80 \%$, las pacientes presentan mejoría del dolor, la menorragia o los síntomas derivados de la compresión pélvica. El tamaño de los miomas, a los dos años de seguimiento, disminuye en un $60-70 \%$. Las complicaciones tardías mas frecuentes serán la infección con emisión vaginal persistente (2\%) y la amenorrea permanente $(2-5 \%)^{21}$.

\section{BIBLIOGRAFÍA}

1. BELCH J. The basic science of angioplasty and restenosis: cellular mechanisms and interventional pharmacotherapy. En: Textbook of endovascular procedures. Dyet JF, Ettles DF, Nicholson AA, Wilson SE. Editores. Churchill Livingstone. Philadelphia 2000: 3-14.

2. PALMAZ JC. Intravascular stents: Tissue-stent interactions and design considerations. AJR 1993; 160: 613-618.

3. CRAGg AH, DAKE MD. Treatment of peripheral vascular disease with stent-grafts. Radiology 1997; 205: 307-314.

4. Vorwerk D, GÜnther R, Schurmann K et al. Primary stent placement for chronic iliac artery occlusions: Follow-up results in 103 patients. Radiology 1995; 194: 745-749.

5. SAnchez LA, Veith F. Femoro-popliteal-tibial occlusive disease. En: Vascular surgery-a comprehensive review. Moore W. Ed. W B Saunders. Philadelphia 1998: 497-520.

6. VARTy K, NydAhl S, NASIM A et al. Results of surgery and angioplasty for treatment of 
chronic severe lower limb ischaemia. Eur J Vasc Surg 1998; 16: 159-163.

7. Blum U, Krumme B, Flugel $P$ et al. Treatment of ostial renal artery stenosis with endovascular endoprosthesis after unsuccessful balloon angioplasty. N Engl J Med 197; 336: 459-465.

8. Matsumoto AH, Tegtmeyer CJ, Fitzcharles CK et al. Percutaneous tansluminal angiolasty of visceral arterial stenoses: Results and long-term clinical follow-up. J Vasc Interv Radiol 1995; 6: 165-174.

9. Theron JG, Payelle GG, Coskun $O$ et al. Carotid artery stenosis: Treatment with protected balloon angioplasty and stent placement. Radiology 1996; 201: 627-636.

10. Turmel-Rodrigues L, Sapoval M, Pengloan J et al. Manual tromboaspiration and dilation of thrombosed dyalisis access: Mid-term results of a simple concept. J Vasc Interv Radiol 1997; 8: 813-824.

11. GaInes P, Kafie FE, WiLSON SE. Upper limb venous and superior vena cava intervention. En: Textbook of endovascular procedures. Dyet JF, Ettles DF, Nicholson AA, Wilson SE. Editores. Churchill Livingstone. Philadelphia 2000: 222-233.

12. PAROdi JC, FERreira LM, Garelli G. Endoprótesis en los aneurismas de aorta abdominal. En: Diagnóstico y Terapéutica Endoluminal. Radiología Intervencionista. Carreira Villamor JM, Maynar Moliner M. Editores. Masson. Barcelona 2002: 294-302.

13. Bilbao Ji, Quiroga J, Herrero JI, Benito A. Transjugular Intrahepatic Portosystemic Shunt (TIPS): Current status and future possibilities. Cardiovasc Intervent Radiol 2002; 25: 251-269.
14. Cosín O Garrido F, Gil $R$, Domínguez $P$ Martínez de la Cuesta A, Bilbao JI. Reservorio intra-arterial hepático para quimioterapia. Colocación percutánea en la arteria subclavia izquierda. Radiología 2005; 47: 329-334.

15. Llovet JM, Real Mi, Montañá X et al. Arterial embolisation or chemoembolisation versus symptomatic treatment in patients with unresectable hepatocellular carcinoma: a randomised controlled trial. Lancet 2002; 18 ; 359:1734-1739.

16. Gimeno MJ, Madariaga B, Alfonso Aguiran ER et al. Hemoptisis amenazante. Tratamiento mediante embolización transcatéter. Arch Bronconeumol 1999; 35: 379-384.

17. Hidalgo F, Narvaez JA, Rene $M$ et al. Treatment of hemobilia with selective hepatic artery embolization. J Vasc Interv Radiol 1995; 6: 793-798.

18. Aina R, Oliva VL, THERASSE E et al. Arterial embolotherapy for upper gastrointestinal hemorrhage: Outcome assessment. J Vasc Interv Radiol 2001; 12: 195-200.

19. FUNAKI B. Superselective embolization of lower gastrointestinal hemorrhage: a new paradigm. Abdom Imaging 2004; 29: 434-438.

20. KIDNEY DD. The endovascular approasch to trauma. En: Textbook of endovascular procedures. Dyet JF, Ettles DF, Nicholson AA, Wilson SE. Editores. Churchill Livingstone. Philadelphia 2000: 313-327.

21. Pinto I, Chimeno P, Romo A et al. Uterine fibroids: uterine artery embolization versus abdominal hysterectomy for treatment-a prospective, randomized, and controlled clinical trial. Radiology 2003; 226: 425-431. 